\title{
A note on the categorization of demand patterns
}

\author{
A.V. Kostenko* and R.J. Hyndman ${ }^{\dagger}$
}

14 February 2006

\begin{abstract}
We revisit the problem of categorizing demand patterns in order to select the best forecasting method. We improve the categorization scheme of Syntetos, Boylan and Croston (2004) by deriving an exact result for the boundary between categories and giving a simple approximation to the boundary that is better than that previously published.
\end{abstract}

Keywords: categorization; forecasting; inventory control; intermittent demand.

The accuracy of a forecasting method for a particular product depends on characteristics exhibited by the product's demand history. Consequently, demand time series are sometimes divided into several discrete categories in order to assign the best forecasting method.

The idea of categorizing demand patterns initially appeared in Williams ${ }^{1}$, who studied the classification of products by demand type, stock control policies for different categories of products, and methods of forecasting demand for the different categories of products. Similar ideas of categorizing demand patterns for the purpose of forecasting and inventory control are considered in Eaves \& Kingsman ${ }^{2}$.

A new approach to this problem was recently provided by Syntetos, Boylan and Croston 3 (hereafter SBC). SBC categorize demand based on the expected mean square error of each forecasting method under some assumptions. They compare Croston's ${ }^{4}$ method (hereafter (RO) and a bias-adjusted version of Croston's method due to Syntetos and Boylan ${ }^{5}$ (also known as the "approximation method"2,6 and hereafter referred to as SBA). From this comparison they propose the four discrete categories of demand shown in Figure 1 which they label 'erratic', 'lumpy', 'smooth' and 'intermittent'. The four quadrants are uniquely specified by two parameters $p$ and $v$, where $p$ is the average inter-demand interval and $v$ is the squared coefficent of variation of the demand when it occurs.

SBC argue that CRO should only be used for smooth demand series and that demand series from the other three quadrants are best forecast using SBA. The threshold values defining the quadrants are given as $p=1.32$ and $v=0.49$ respectively.

Both CRO and SBA use a smoothing constant $\alpha$ for producing exponentially smoothed ${ }^{7}$ estimates of positive demands. They also both use the parameter $p$ to denote the average inter-demand interval. The parameter $\alpha$ is usually restricted to the range $(0,1)$ and $p \geq 1$ by definition.

${ }^{*}$ A.V. Kostenko, Independent Complex Systems Consultant, 665714, Bratsk, Russia. All correspondence to admin@ruenglish.com

${ }^{\dagger}$ R.J. Hyndman, Department of Econometrics \& Business Statistics, Monash University VIC 3800, Australia. 


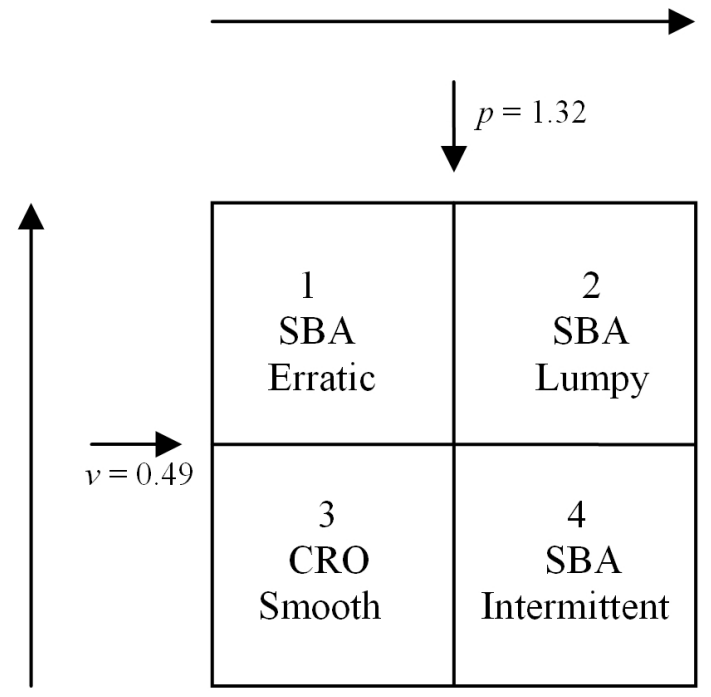

Figure 1: The SBC categorization scheme for $C R O$ and SBA methods for forecasting intermittent demand data.

Assuming that demand data are independent and identically distributed, SBC showed that the theoretical mean squared error (MSE) of CRO was greater than that of SBA when

$$
v>\frac{(p-1)\left[\frac{4(p-1)}{(2-\alpha)}-\frac{2-\alpha}{p-1}-p(\alpha-4)\right]}{\frac{p(\alpha-4)(2 p-\alpha)}{2-\alpha}} .
$$

(This is equation (8) in SBC.)

We simplify (1) by rewriting it as

$$
v>\frac{4 p(2-p)-\alpha(4-\alpha)-p(p-1)(4-\alpha)(2-\alpha)}{p(4-\alpha)(2 p-\alpha)}
$$

This defines the region of the parameter space where SBA produces better forecasts than CRO. Figure 2 shows the boundary of the region for several values of $\alpha$. Note that this graph corresponds to the 'Smooth' region of Figure 1.

The limiting value of $p$ is obtained when $v=0$ and $\alpha=0$ giving $p=4 / 3$ (not 1.32 as given by $\mathrm{SBC}$ ). This exact value of $p$ is a solution of the following equation:

$$
0=\frac{\frac{1}{2} p(2-p)-p(p-1)}{p^{2}}
$$

When $p=1$ and $\alpha=0$, we find the maximum value of $v=0.5$ (not 0.49 as given by SBC) for which CRO can be better than SBA.

SBC propose using CRO for all values of $p$ and $v$ shown in Figure 2. However, for most of this region, SBA is actually more accurate. Furthermore, Figure 2 shows that there are only two demand categories ('smooth' and 'lumpy') that can be associated with these two methods of forecasting intermittent demand. To summarize: when using the CRO and SBA methods, use SBA unless $p$ and $v$ fall below the line in Figure 2. 


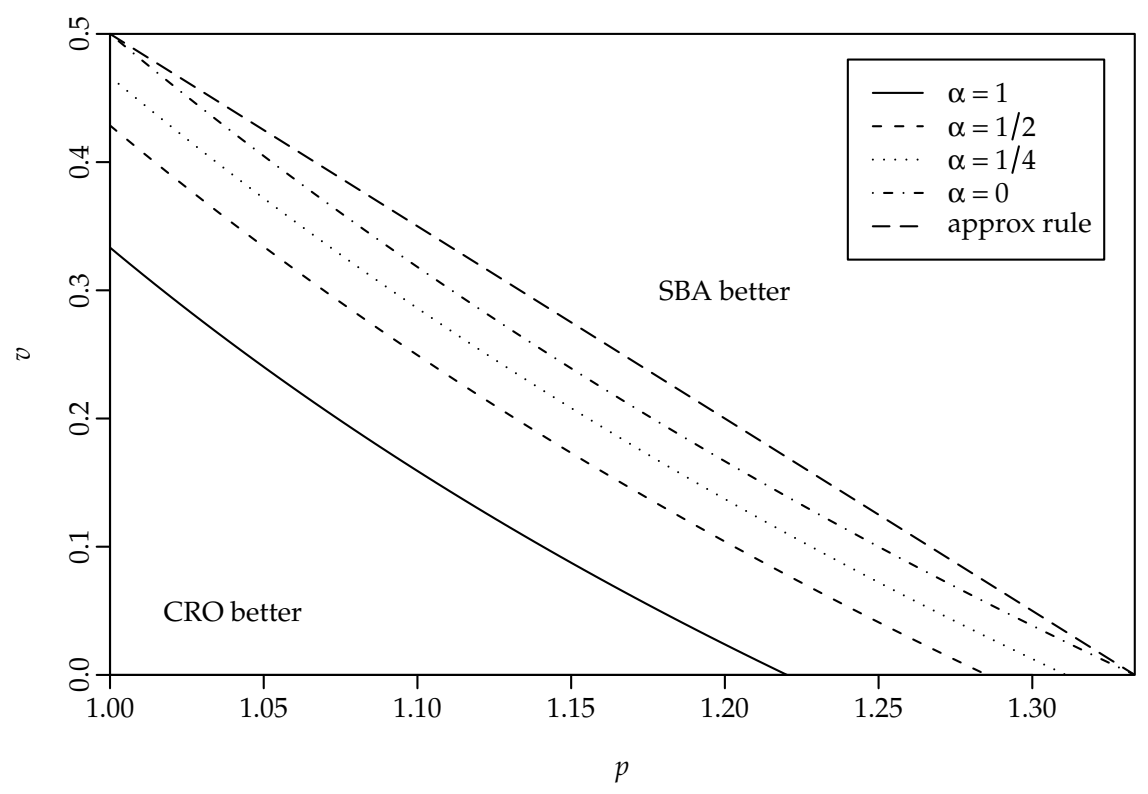

Figure 2: Parameter space for which each forecasting method is better than the other.

Possibly SBC used their four-quadrant approach to enable a simple rule to be implemented. We suggest that a simple but more accurate rule can be derived by dividing the quadrant diagonally in two. In this case, the rule is to use SBA whenever $v>2-\frac{3}{2} p$. This approximation is also shown in Figure 2. Because $\alpha$ is usually small (less than 0.3) for intermittent demand data, this approximation is quite good. In any case, the comparative advantage of one method over the other in the space between the approximate rule and the true boundary will be small.

One of the assumptions underlying the SBC analysis (and Croston's original analysis ${ }^{4}$ ) is that it assumes that the demand values are independent. This is clearly never true, and if it was true we would simply use the historical mean as the best predictor rather than any more sophisticated forecasting method. In fact, it has recently been shown ${ }^{8}$ that Croston's method (as originally proposed in 1972) and related methods cannot be optimal for any time series of demand, regardless of their time series properties. Consequently, we suggest that a more useful line of research may be to find forecasting methods that are optimal assuming realistic properties of demand time series. 


\section{References}

1 Williams TM (1984) Stock control with sporadic and slow moving demand. J Opl Res Soc 35: 939-948.

2 Eaves AHS and Kingsman BG (2004) Forecasting for the ordering and stock-holding of spare parts. J Opl Res Soc 55: 431-437.

3 Syntetos AA, Boylan JE and Croston JD (2005) On the categorization of demand patterns. J Opl Res Soc 56: 495-503.

4 Croston JD (1972) Forecasting and stock control for intermittent demands. Opl Res Q 23: 289-304.

5 Syntetos AA and Boylan JE (1999) Correcting the bias of forecasts of intermittent demand. Presented at the 19th International Symposium on Forecasting, June 1999, Washington DC, USA.

6 Syntetos AA (2001) Forecasting of intermittent demand. Unpublished PhD Thesis, Buckinghamshire Business School, Brunel University, UK.

7 Makridakis S, Wheelwright SC and Hyndman RJ (1998) Forecasting: methods and applications, 3rd ed., John Wiley \& Sons: New York.

8 Shenstone L and Hyndman RJ (2005) Stochastic models underlying Croston's method for intermittent demand forecasting. J of Forecasting, 24: 389-402. 